\title{
A Novel Wireless Sensor Network Evolution Model Based on Energy-Efficiency
}

\author{
https://doi.org/10.3991/ijoe.v13i03.6855 \\ Hui Gao* \\ Zhengzhou Railway Vocational \& Technical College, Zhengzhou City, China \\ gaohui $489 @$ sohu.com \\ Zhixian Yang \\ Zhengzhou Railway Vocational \& Technical College, Zhengzhou City, China \\ yzx_2004@126.com
}

\begin{abstract}
The Barabási-Albert (BA) model is a famous complex network model that generates scale-free networks. Wireless sensor networks (WSNs) had been thought to be approximately scale-free through lots of empirical research. Based on the BA model, we propose an evolution model for WSNs. According to actual influence factors such as the remainder energy of each sensor and physical link capability of each sensor, our evolution model constructs WSNs by using a preferential attachment mechanism. Through simulation and analysis, we can prove that our evolution model would make the total energy consumption of the WSNs more efficient and have a superior random node error tolerance.
\end{abstract}

Keywords-Wireless sensor network; Complex networks; Evolution model; Energy-aware; Energy-balanced

\section{Introduction}

WSNs, which are usually called wireless sensor and actuator networks (WSANs) [1], are remotely located wireless sensors to monitor realistic conditions, and to bidirectionally transfer information by the networks to a master position. The improvement of WSNs originated in some special applications such as natural disaster relief. Now WSNs are used widely in many societal fields, such as industry supervisory control, machinery equipment performance monitoring, traffic control, and so on [2, $3]$.

WSNs always are made up of "sensor nodes" - from several hundreds to even tens of thousands, where each sensor node is connected with other sensor nodes in the same network. Each such node consists of several parts: a radio transceiver with an internal antenna, a microcontroller, an electronic circuit, and a limited power energy battery source. Because they are battery-operated and remotely-deployed, once deployed, the frequent battery replenishment of sensors is unrealistic. Hence, how to use 
the limited energy to effectively balance the energy consumption of each sensor and lengthen the network life cycle is a crucial problem when it comes to WSNs.

There are many systems which can be described in terms of complex networks in nature and society [4], such as online social networks [5,6], protein-protein interaction networks [7], the Internet network [8,9], and transportation networks [10,11]; therefore, experts have paid a considerable amount of attention to the topology and dynamic nature of complex networks in various domains.

Notably, Barabasi and Albert have proposed the scale-free model, which is a dynamic evolution model to explain the phenomenon of power-law degree distribution; this distribution is called "preferential attachment" [12, 13]. A scale-free network is a network whose degree of nodes follows the power-law distribution. This means, the fraction $P(k)$ of vertexes in the network having $k$ links to other nodes goes for large values of $k$ as $P(k) \sim k^{-\gamma}$, where $\gamma$ is a variable whose value is representatively in the scope $2<\gamma<3$. Preferential attachment and the corresponding model [14] have been proposed as mechanisms to clarify the power-law distributions of node degrees in actual networks.

There have been lots of distributed topology control algorithms based on energybalance and energy-awareness for WSNs over the past few years. Mohite [15] proposed an energy-efficient path-finding solution for related data collection in WSNs. Huang et al., [16] presented a variety of theoretical studies on $k$-covered WSNs based on clustering algorithms. Kumar and Ahlawat [17] proposed a new communication topology evolution approach between clustering center nodes to reduce the energy consumption of WSNs. Wang et al., [18] introduced several topology control algorithms which were used to maintain the connectivity and coverage of WSNs. Miyao et al., [19] focused mainly on minimizing energy consumption and ensuring fault tolerance. But nearly all existing research has failed to study the energy efficiency in WSNs from the topology structure and vertex degree distribution point of view based on complex networks.

In this paper, an evolution model for WSNs based on complex networks is proposed. The evolution model is based on energy-awareness and energy-balance, its generation method is based on preferential attachment, and it will generate scale-free networks which have the advanced capability of resisting random errors. Moreover, the maximum number of edges for each sensor vertex will be limited to prevent highdegree nodes from consuming energy excessively.

This paper is organized as follows. An evolution model considering the energyawareness of sensor nodes and energy balance of the whole network based on a scalefree model is proposed in Section 2. In Section 3, experiments and an analysis are given to present the characteristics of the networks generated by our model. Finally, Section 4 presents the conclusions of our paper. 


\section{Evolution Model of Wireless Sensor Networks for Communication Topology}

Although WSNs are a sort of typical complex network, they have their own special characteristics. Therefore, to achieve the uniform distribution of energy consumption in WSNs, actual factors such as transmission radius of the nodes, energy efficiency, and the constraint of the maximum links' number of each node should be considered.

In WSNs, all nodes spend most of their energy during the data transfer phase after the completion of network organization, and the energy consumption of each node may vary, so we should assume that the remainder energy of every sensor node is different and fixed. Besides, each sensor node in WSNs can only connect to adjacent vertexes which are located in its transfer scope under the restriction of the sensor node transmission radius. This results in a very small amount of neighbor vertexes per node. Through plenty of empirical studies at home and abroad [20-30], we can confirm that there is a scale-free structure in WSNs. However, there are a lot of highdegree vertexes in scale-free networks; these sensor nodes need to connect to a large number of nodes, so they will exhaust energy fast. Moreover, we should try to prevent the emergence of such nodes in the actual network. So in our evolution model, we assume that each sensor node can connect to no more than its maximum number of connections, and the maximum number of connections also depends on its remainder energy.

The important parameters of our evolution model are shown in Table 1.

Table 1. Important parameters of our evolution model

\begin{tabular}{|c|c|}
\hline Parameters & Definition in Wireless Sensor Networks \\
\hline$m_{0}$ & Initial number of nodes in initial network \\
\hline$m$ & Number of new links connected to a new vertex per time interval \\
\hline$k_{i}$ & Number of edges connected with vertex $i$ \\
\hline$R$ & Remainder energy of a sensor vertex \\
\hline$k_{i}$ & $\begin{array}{l}\text { Probability of a just coming sensor vertex which would be con- } \\
\text { nected with vertex } i\end{array}$ \\
\hline$\rho$ & Distribution of the remainder energy of all the vertexes \\
\hline$S$ & Number of nodes within each new coming node's local scope \\
\hline$t_{i}$ & Time of vertex $i$ just coming into the evolution network \\
\hline
\end{tabular}

The generation process of a WSN by our model is as follows:

(1) Growth: The initial number of nodes to be determined is $m_{0}$, within each time step, a new coming vertex with $m\left(m<m_{0}\right)$ edges which would be linked to the vertexes already existing in the initial network is added.

(2) Preferential attachment: As a new coming node is added to the existing network, some candidate vertexes in its local-scope will be selected to connect. The probability $\prod_{i} k_{i}$ of a new coming vertex being attached to vertex $i$ depends on the connectivity $k_{i}$, and its remainder energy $R$ of the node. A correlative function $f(R)$ is defined to represent such a relationship between a node's remainder energy and its 
ability to be connected. We assume that the more remainder energy a vertex has, the stronger the ability of a vertex which would be connected to the new coming vertexes. So function $f(R)$ should be a growth function, the form of function $f(R)$ may be such as a $\sqrt{R}, b \log _{2} R$ and so on.

The probability of preferential attachment $\prod_{i} k_{i}$ is defined as

$$
\prod_{i} k_{i}=\frac{f(R) k_{i}}{\sum_{j \in \text { local-scope }} f\left(R_{j}\right) k_{j}}
$$

According to Growth, during each time step, $m$ new links are formed. So the following formula can be obtained:

$$
\frac{\partial\left(k_{i}\right)}{\partial(t)} \approx m \prod_{i} k_{i}=m \frac{f(R) k_{i}}{\sum_{j \in \text { local }- \text { scope }} f\left(R_{j}\right) k_{j}}
$$

In the local-scope of each sensor node, the following formula can be obtained:

$$
\sum_{j \in l o c a l-s c o p e} f\left(R_{j}\right) k_{j}=S \bar{R}\langle k\rangle
$$

where $S$ is the number of vertexes within the new coming vertex's local-scope, $\bar{R}$ is the mathematical expectation of $f(R)$, and $\langle k\rangle$ is the average degree of all nodes. In complex networks, the average degree can be represented as follows:

$$
\langle k\rangle=\frac{2\left(m t+e_{0}\right)}{m_{0}=t} \approx 2 m
$$

where $e_{0}$ and $m_{0}$ represent individually the number of edges and vertexes of the initial network, and their value should be very small.

From Eqs. (2), (3) and (4), we can get the following formula:

$$
\frac{\partial\left(k_{i}\right)}{\partial(t)}=\frac{f(R) k_{i}}{2 S \bar{R}}
$$

So we can conclude

$$
\frac{\partial\left(k_{i}\right)}{k_{i}}=\frac{f(R) k_{i}}{2 S \bar{R}} d t
$$


Because $f(R)$ is a growth function, later, we will show that the expression of $f(R)$ actually does not influence the final degree distribution. We can assume that if we set $f(R)=R$, then

$$
k_{i}=e^{\frac{R}{2 S \bar{R}} t+C}
$$

Because of $k_{i}\left(t_{i}\right)=m$, then $\mathrm{C}=\ln m-\frac{R}{2 S \bar{R}} t_{i}$, then we can conclude

$$
k_{i}=e^{\frac{R}{2 S \bar{R}}\left(t-t_{i}\right)} \times m
$$

The probability of a node whose connectivity is $k_{i}(\mathrm{t}) \leq k$ could be represented as

$$
P\left(k_{i}(t)<k\right)=P\left(\frac{R}{2 S \bar{R}}\left(t-t_{i}\right)<\ln \left(\frac{k}{m}\right)\right)=P\left(t-\frac{2 S \bar{R}}{R} \ln \left(\frac{k}{m}\right)<t_{i}\right)
$$

We suppose that if the new coming vertex is attached to the network at regular intervals, then the probability density at the time $t_{i}$ is $P_{i}\left(t_{i}\right)$

$$
P_{i}\left(t_{i}\right)=\frac{1}{m_{0}+t}
$$

From Eqs. (9) and (10), we can conclude

$$
P\left(k_{i}(t)<k\right)=1-P\left(t_{i} \leq t-\frac{2 S \bar{R}}{R} \ln \left(\frac{k}{m}\right)\right)=1-\frac{t-\frac{2 S \bar{R}}{R} \ln \left(\frac{k}{m}\right)}{m_{0}+t}
$$

So the probability density function of a vertex with remainder energy $R$ can be expressed as

$$
P\left(k_{R}\right)=\frac{\partial P\left(k_{i}(t)<k\right)}{\partial k}=\frac{1}{m_{0}+t} \frac{2 S \bar{R}}{R} \frac{m}{k}
$$

For achieving the probability density function of the whole network, a weighted average of all nodes' probability density functions is taken as the probabilities of having remainder energy $R$. So $k$ acts as a continuous random variable for the degree of the vertex. 


$$
P(k)=\int_{R_{\min }}^{R_{\max }} \rho P\left(k_{R}\right) d R=\int_{R_{\min }}^{R_{\max }} \rho \frac{1}{m_{0}+t} \frac{2 S \bar{R}}{R} \frac{m}{k} d R
$$

where $\rho$ is the distribution of $R, E_{\max }$ and $E_{\min }$ are the maximum and minimum values of the remainder energy, respectively.

It is obvious that $P(k)$ obeys $P(k) \propto \lambda k^{-1}$, where

$$
\lambda=\int_{R_{\min }}^{R_{\max }} \rho \frac{1}{m_{0}+t} \frac{2 S \bar{R}}{R} d R
$$

From Eq. (14), we can see that $k$ is a constant in fact for a given network.

Thus, our evolution model can generate WSNs with scale-free characteristics in an energy-aware way. But in scale-free networks, there will be some vertexes which may connect to many other vertexes. This will result in the rapid depletion of the energy of these nodes. In consideration of the energy-balance of the whole network, we need to modify our model to prevent the vertexes from connecting with too many other vertexes.

In a modified model, we assume that each vertex can only connect with no more than $k_{i(\max )}$ vertexes, and the value of $k_{i(\max )}$ depends on the vertex's remainder energy $R$. Let us suppose that a node whose maximum energy is $R_{\max }$ can connect with no more than $k_{\max }$ nodes. We can conclude

$$
k_{i(\max )}=k_{\max } \frac{R}{R_{\max }}
$$

The generation process of a WSN by the modified evolution model after introducing the limitation of maximum node degree is as follows:

(1) Growth: The initial number of nodes to be determined is $m_{0}$, within each time step, a new coming vertex with $m\left(m<m_{0}\right)$ links which would be connected to the vertexes already existing in the network is added.

(2) Preferential attachment: As a new vertex is added to the existing network, some candidate vertexes in its local-scope will be selected to connect.. The probability $\prod_{i} k_{i}$ that a new coming vertex would be attached to vertex $i$ depends on the connectivity $k_{i}$, and its remainder energy $R$ of the node. At the same time, node $i$ could only be connected with no more than $k_{i(\max )}$ nodes.

The probability of the preferential attachment $\prod_{i} k_{i}$ is defined as 


$$
\prod_{i} k_{i}=\frac{f\left(R, k_{i}\right) k_{i}}{\sum_{j \in \text { local-scope }} f\left(R_{j}, k_{j}\right) k_{j}}
$$

where $\left(R, k_{i}\right)=R\left(1-\frac{k_{i}}{k_{i(\max )}}\right)$, and $k_{i(\max )}=k_{\max } \frac{R}{R_{\max }}, 1-\frac{k_{i}}{k_{i(\max )}}$ means that the closer $k_{i}$ is to $k_{i(\max )}$, the lower the probability the vertex will be chosen to connect with the new coming vertexes. If $k_{i}$ has reached its maximum degree $k_{i(\max )}$, the node $i$ would not be chosen to connect with the new coming nodes forever.

Like with the previous evolution model, we can get

$$
\frac{\partial\left(k_{i}\right)}{\partial(t)} \approx m \prod_{i} k_{i}=m \frac{f\left(R, k_{i}\right) k_{i}}{\sum_{j \in \text { local }- \text { scope }} f\left(R_{j}, k_{j}\right) k_{j}}
$$

Because most nodes' degree $k_{i}$ is far less than their maximum, namely, $k_{i} \ll$ $k_{i(\max )}$, we can conclude

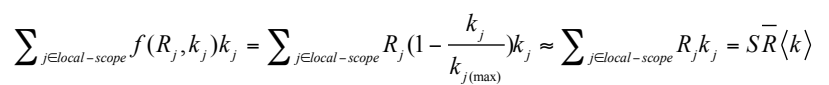

where $\langle k\rangle=\frac{2\left(m t+e_{0}\right)}{m_{0}+t} \approx 2 m$, and the following formula can be obtained:

$$
k_{i}=\frac{k_{i(\max )}}{\frac{k_{i(\max )}-m}{m} e^{\frac{R}{2 S \bar{R}}\left(t_{i}-t\right)}+1}
$$

Similarly, $P(k)$ of the modified evolution model can be represented as follows:

$$
P(k)=\int_{R_{\min }}^{R_{\max }} \rho P\left(k_{R}\right) d R=\int_{R_{\min }}^{R_{\max }} \rho \frac{2 S \bar{R}}{m_{0}+t} \frac{k_{\max }}{R_{\max }} \frac{1}{k k_{i(\max )}-k^{2}} d R
$$

where $\rho$ is the distribution of $R, k$ acts as a successive random variable for the degree of the vertex, and $E_{\max }$ and $E_{\min }$ are the maximum and minimum values of the remainder energy, respectively.

In the modified evolution model, $P(k)$ can be functional, and it will vary with various energy distributions. The topology structure of the network generated by the modified evolution model may only be approximately scale-free, 


\section{Experiments and Analysis}

In the course of an experiment, we suppose that the initial network begins with 5 nodes, namely $m_{0}=5$. At each time step, a new coming vertex is connected with 3 existing vertexes, thus $m=3$. For the evolution network, we suppose that the remainder energy of each vertex varies from $0.5 \mathrm{~J}$ to $1 \mathrm{~J}$, and the corresponding parameter of the model is $0.5 \leq R \leq 1$.

The distribution of the remainder energy is critical to the final generated network. Three various distributions of remainder energy are given in experiments. Table 2 shows the distribution functions of and corresponding mathematical expectations for the remainder energy.

Table 2. Different distribution of remainder energy

\begin{tabular}{|c|c|}
\hline Distribution of remainder energy $R$ & Mathematical expectation for remainder energy \\
\hline Distribution of uniform, $p(R)=2$ & 0.75 \\
\hline Distribution of power-law, $p(R) \sim R^{-1}$ & 0.5 \\
\hline Distribution of power-law, $p(R) \sim R^{-3}$ & 1 \\
\hline
\end{tabular}

In order to validate the capability of the generated networks with our evolution model, we analyze the evolution results with the connectivity degree distribution. The effect of energy distribution on the connectivity growth of nodes is shown in Figure 1. With different degree distributions, the different mathematical expectations would generate various speeds of connectivity growth.

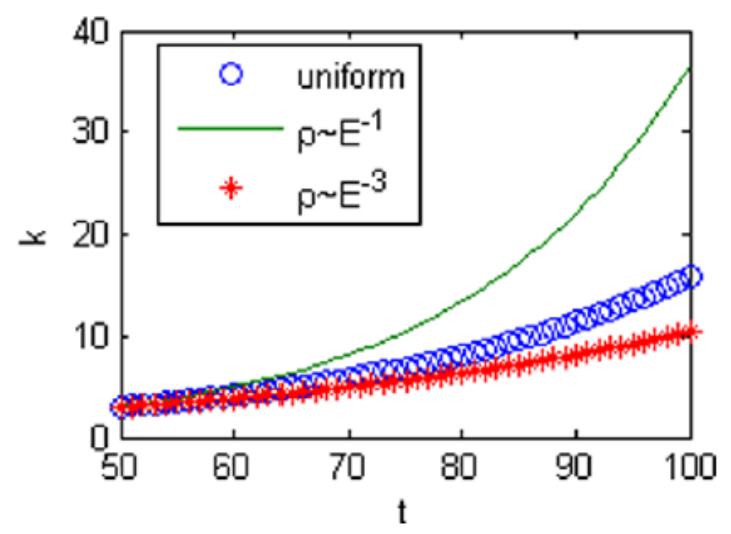

Fig. 1. Degree of evolution network with different energy distributions

Parameters of the model are $R=1, S=20, t_{i}=50$, and $m=3$ in Figure 1.

From Figures 2 and 3, we can see how the local-scope and vertexes' incoming time respectively affect the node degree. 


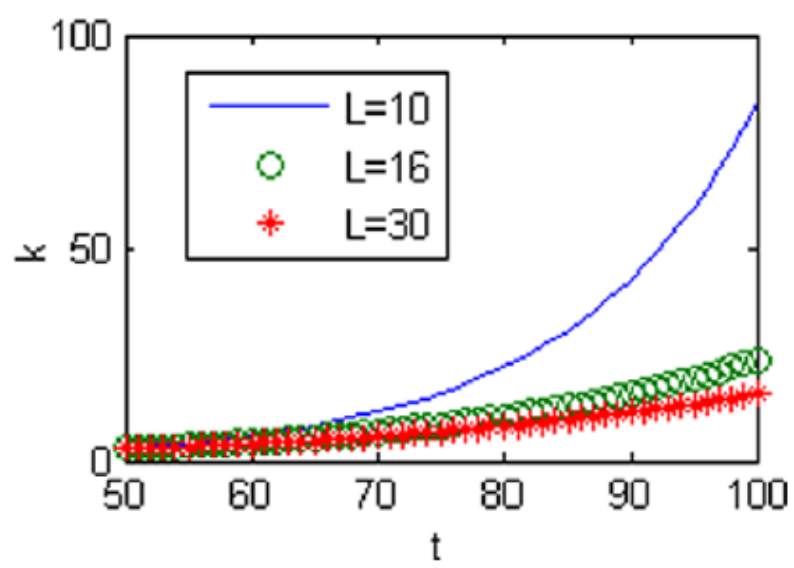

Fig. 2. Degree of evolution network with different local-scopes

Parameters of the model are $R=1, t_{i}=50$, and $m=3$ in Figure 2 .

Figure 2 shows that the newly coming nodes would select various numbers of vertexes based on the range of the local-scope. We can see that the smaller $S$ is, the higher the probability of the node $i$ to be connected.

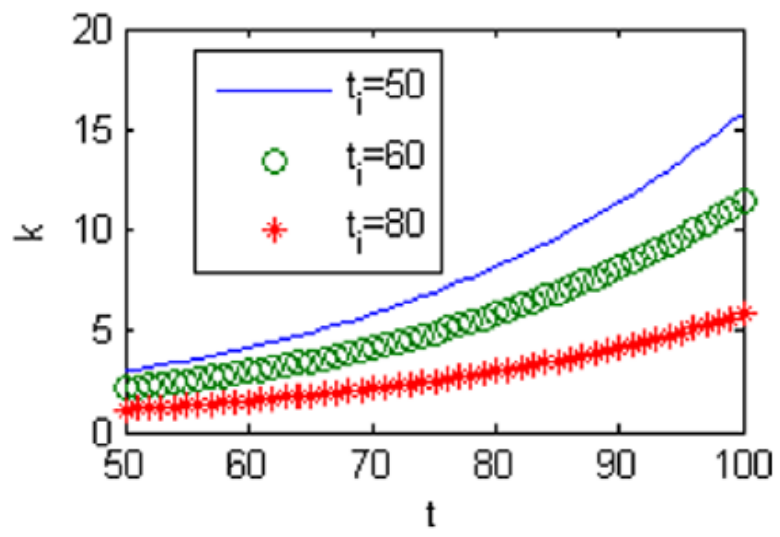

Fig. 3. Degree of evolution network with different vertex incoming times

Parameters of the model are $R=1, S=20$, and $m=3$ in Figure 3 .

Figure 3 indicates the effect of a new node's coming time on the connectivity growth. From Figure 3, we can see that the older vertexes have a greater chance of being selected for linking to the new coming vertexes, which means that the connectivity of the older vertexes would increase more rapidly than that of the younger.

The degree distribution of the generated networks by our evolution model with various energy distributions is shown in Figure 4. 


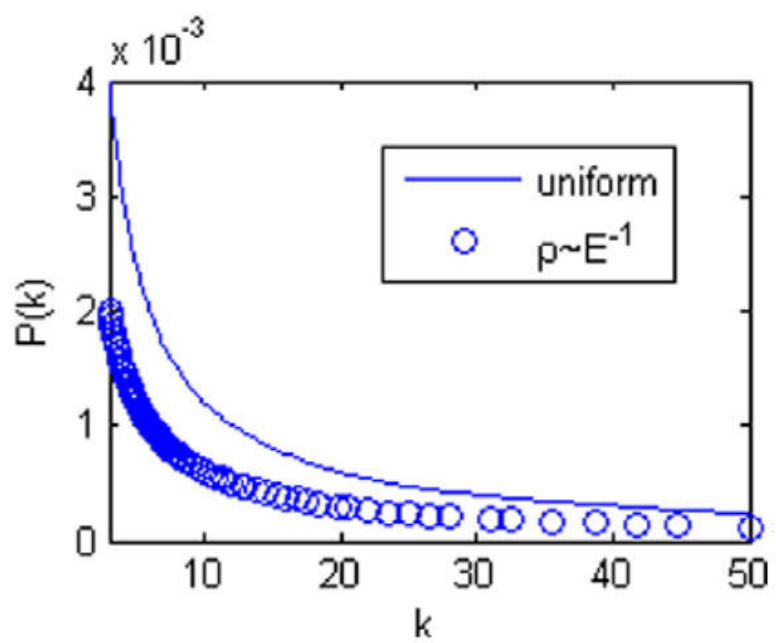

Fig. 4. Degree distribution with various energy distributions

Parameters of the model are $m_{0}=5, S=10$, and $m=3$ in Figure 4 .

From Figure 4, we can see that the degree distribution follows the power-law distribution despite the distribution of the remainder energy. This means that scale-free WSNs can be obtained by our evolution model in any case.

The above experiments do not take the maximum connectivity constraints $k_{i(\max )}$ into consideration. After adding the factor into the model, the degree distribution with various energy distributions and the degree of the evolution network with different local-scopes are shown respectively in Figures 5 and 6.

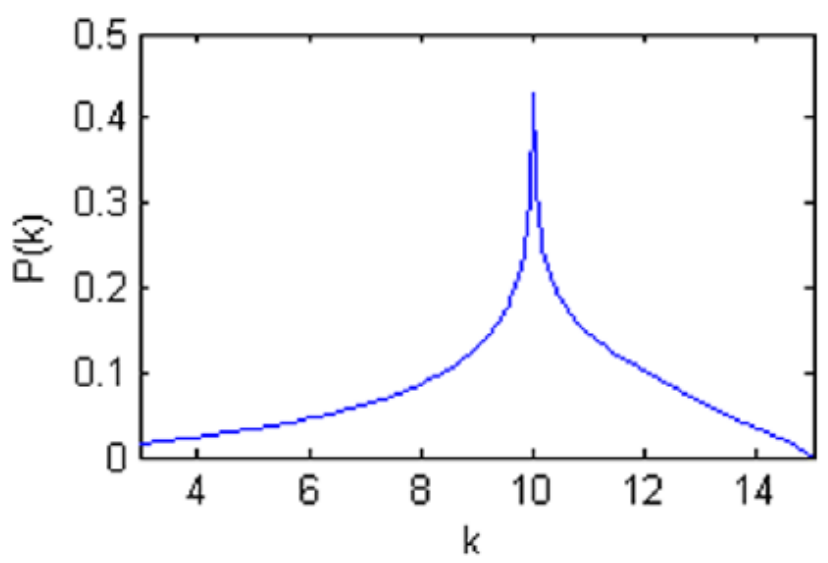

Fig. 5. Degree distribution with uniform energy distributions 


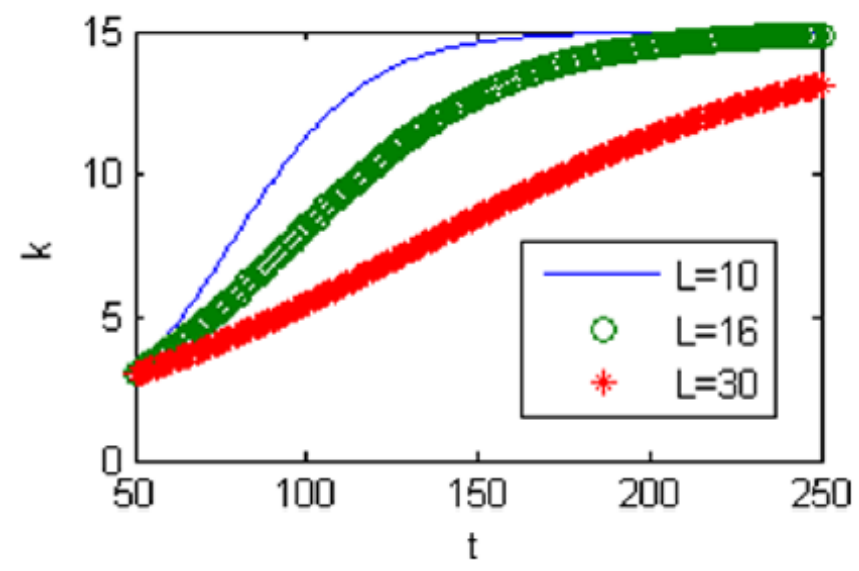

Fig. 6. Degree of network with different local-scopes in modified model

From Figures 5 and 6 , we can see that the connectivity speed is much slower because of the maximum connectivity constraints.

\section{Conclusions}

We have proposed an evolution model for revealing truly the realistic WSNs in this paper. In our model, we added new nodes and links based on the energy-awareness of each sensor node and the energy-balance of the whole network. The simulation results in our experiment have shown that our model is highly compatible with the actual network topology with regards to degree distribution and other critical topologyrelated metrics.

\section{$5 \quad$ References}

[1] Zhang, C. (2010). Cluster-based Routing Algorithms Using Spatial Data Correlation for Wireless Sensor Networks. Journal of Communications, 5(3): 232-238. https://doi.org/10.4304/jcm.5.3.232-238

[2] Wang, J., Kim, J. U., Shu, L. (2010). A Distance-based Energy Aware Routing algorithm for wireless sensor networks. Sensors, 10(10): 9493-9499. https://doi.org/10.3390/ s101009493

[3] Zhang, D. (2016). Heating Meter Reading System Based on GPRS and Radio Frequency Technology. Revista de la Facultad de Ingeniería, 31(9): 177-185.

[4] Fichera A., Fortuna L., Frasca M., Volpe R. (2015). Integration of complex networks for urban energy mapping, International Journal of Heat and Technology, 33(4), 181-184. https://doi.org/10.18280/ijht.330423

[5] Sun, G., Sheng, B., Zhou, Y. (2015). Big Data Analytics of Multi-Relationship Online Social Network Based on Multi-Subnet Composited Complex Network. International Journal of Database Theory \& Application, 8(5): 273-284. https://doi.org/10.14257/ijd ta.2015.8.5.24 
[6] Zhang, W., Sun, G., Sheng, B. (2015). An Opinion Leaders Detecting Algorithm in Multirelationship Online Social Networks. International Journal of Hybrid Information Technology, 9(5): 391-398. https://doi.org/10.14257/ijhit.2016.9.5.33

[7] Chua, H. N., Ning, K., Sung, W. K. (2011). Using indirect protein-protein interactions for protein complex prediction. Computational Systems Bioinformatics, 6(3): 97-109.

[8] Sánchezburillo, E., Duch, J., Zueco, D. (2012). Quantum Navigation and Ranking in Complex Networks. Scientific Reports, 2: 605-616.

[9] Paparo, G. D., Müller, M., Comellas, F. (2013). Quantum Google in a Complex Network. Scientific Reports, 3: 127-132. https://doi.org/10.1038/srep02773

[10] Tsiotas, D., Polyzos, S. (2015). Decomposing multilayer transportation networks using complex network analysis: a case study for the Greek aviation network. Journal of Complex Networks, 3(4): 624-670. https://doi.org/10.1093/comnet/cnv003

[11] Shi, H. S. (2016). A Method of Automatic Detection of Fog Image Based on SVM Classification. Revista de la Facultad de Ingeniería, 31(9): 211-218.

[12] Barabasi, A., Albert, R. (1999). Emergence of scaling in random networks. Science, 286: 509-512. https://doi.org/10.1126/science.286.5439.509

[13] Barabasi, A., Albert, R. (1999). Mean-field theory for scale-free random networks. Phys Rev A, 272(1-2): 173-187. https://doi.org/10.1016/s0378-4371(99)00291-5

[14] Clauset, A., Shalizi, C. R., Newman, J. (2009). Power-Law Distributions in Empirical Data. Siam Review, 4: 661-703. https://doi.org/10.1137/070710111

[15] Mohite, P. (2015). Adaptive Data Fusion for Energy Efficient Routing in Wireless Sensor Network. International Journal of Energy Optimization \& Engineering, 4(1): 1-17. https://doi.org/10.4018/ijeoe.2015010101

[16] Huang, H. P., Chen, J. T., Wang, R. C. (2014). Compressed Sensing Algorithm Based on Data Fusion Tree in Wireless Sensor Networks. Dianzi Yu Xinxi Xuebao/journal of Electronics \& Information Technology, 36(10): 2364-2369.

[17] Kumar, L., Ahlawat, M. (2012). Evolution Techniques for Reliability of Wireless Sensor Network. International Journal of Advances in Engineering Sciences, 4: 77-87.

[18] Wang, Q., Chen, J., Fang, H. (2014). Fault-tolerant topology control algorithm for mobile robotic networks. International Journal of Control, Automation and Systems, 12(3): 582589. https://doi.org/10.1007/s12555-013-0195-5

[19] Miyao, K., Nakayama, H., Ansari, N. (2010). LTRT: An efficient and reliable topology control algorithm for ad-hoc networks, IEEE Transactions on, 12: 6050-6058.

[20] Konstantinidis, A., Yang, K., Chen, H. H. (2007). Energy-aware topology control for wireless sensor networks using memetic algorithms, Computer Communications, 30(14-15): 2753-2764. https://doi.org/10.1016/j.comcom.2007.05.013

[21] Sehaba, K., Serna, A. (2014). Decoding Social Situations in Adolescents with Asperger through a Serious Game, Modelling, Measurement and Control C, 75(2): 234-247.

[22] Semache A., Hamidat A., Benchatti A. (2015). Impact study of the solar energy on the energy performances of the rural housing in Algeria, International Journal of Heat and Technology, 33(4), 229-236. https://doi.org/10.18280/ijht.330431

[23] Ferentinos, K. P., Tsiligiridis, T. A. (2010). A memetic algorithm for optimal dynamic design of wireless sensor networks, Computer Communications, 2: 250-258. https://doi.org/10.1016/j.comcom.2009.09.004

[24] Liu, H. R., Yin, W. X., Dong, M. R. (2014). Study on the scale-free topology model with strong intrusion-tolerance ability in wireless sensor networks, Acta Physica Sinica, 63(9): 972-986.

[25] Zhou, J., Guo, K., Ye, S. (2015). The review of error-eliminating theories, Advances in Modelling and Analysis A, 52(1): 1-16. 
[26] Genco A., Viggiano A., Viscido L., Sellitto G., Magi V. (2016). Numerical simulation of energy systems to control environment microclimate, International Journal of Heat and Technology, 33(S2), S545-S552. https://doi.org/10.18280/ijht.34S249

[27] Liu, H., Yin, R., Liu, B. (2016). A scale-free topology model with fault-tolerance and intrusion-tolerance in wireless sensor networks, Computers \& Electrical Engineering, 56: 533-543. https://doi.org/10.1016/j.compeleceng.2016.01.003

[28] Trancossi M., Pascoa J.C., Xisto C.M. (2016). Design of an innovative off road hybrid vehicle by energy efficiency criteria, International Journal of Heat and Technology, 33(S2), S387-S395. https://doi.org/10.18280/ijht.34S228

[29] Chekroun, S., Abdelhadi, B., Benoudjit, A. (2014). A New Approach Design Optimizer of Induction Motor Using Particle Swarm Algorithm, Modelling, Measurement and Control A, 87(2): 89-108.

[30] Puglisi G., Zanghirella F., Ungaro P., Cammarata G. (2016). A methodology for the generation of energy consumption profiles in the residential sector, International Journal of Heat and Technology, 33(3), 491-497. https://doi.org/10.18280/ijht.340320

\section{Authors}

Hui Gao, majoring in computer application technology, master of engineering, engaged in computer teaching and network $\mathrm{R} \& \mathrm{D}$, with research focused on computer network and application. He once participated in the national key teacher training and college key laboratory construction and also participated in the provincial project Research on Application of Semiconductor Nanowires in Solar Cells, which was identified as one of the scientific and technological achievements in Henan Province.

Zhixian Yang, majoring in computer application technology, master of engineering, engaged in computer teaching and software R\&D, with research focused on computer and IOT application.

Submitted 02 February 2017. Published as resubmitted by the authors 09 March 2017. 